\title{
Near-conformal dynamics in a chirally broken system
}

\author{
Thomas Appelquist, ${ }^{1}$ Richard C. Brower, ${ }^{2}$ Kimmy K. Cushman $\odot,{ }^{1}$ George T. Fleming, ${ }^{1}$ Andrew D. Gasbarro, ${ }^{3}$ \\ Anna Hasenfratz $\odot,{ }^{4}$ Xiao-Yong Jin $\odot,{ }^{5}$ Ethan T. Neil $\odot,{ }^{4}$ James C. Osborn $\odot,{ }^{5}$ Claudio Rebbi $\odot,{ }^{2}$ \\ Enrico Rinaldi॰, ${ }^{6}$ David Schaich $\odot,{ }^{7}$ Pavlos Vranas $\odot,{ }^{8,9}$ and Oliver Witzel $\odot^{4, *, \dagger}$ \\ (Lattice Strong Dynamics Collaboration)

\begin{abstract}
${ }^{1}$ Department of Physics, Sloane Laboratory, Yale University, New Haven, Connecticut 06520, USA
${ }^{2}$ Department of Physics and Center for Computational Science, Boston University, Boston, Massachusetts 02215, USA

${ }^{3}$ Institute for Theoretical Physics, University of Bern, 3012 Bern, Switzerland

${ }^{4}$ Department of Physics, University of Colorado, Boulder, Colorado 80309, USA

${ }^{5}$ Leadership Computing Facility, Argonne National Laboratory, Argonne, Illinois 60439, USA

${ }^{6}$ Arithmer Inc., R\&D Headquarters, Minato, Tokyo 106-6040, Japan and Interdisciplinary Theoretical and

Mathematical Sciences Program (iTHEMS), RIKEN, 2-1 Hirosawa, Wako, Saitama 351-0198, Japan

${ }^{7}$ Department of Mathematical Sciences, University of Liverpool, Liverpool L69 7ZL, United Kingdom

${ }^{8}$ Nuclear and Chemical Sciences Division, Lawrence Livermore National Laboratory, Livermore, California 94550, USA
\end{abstract} \\ ${ }^{9}$ Nuclear Science Division, Lawrence Berkeley National Laboratory, Berkeley, California 94720, USA
}

(Received 16 July 2020; accepted 15 December 2020; published 5 January 2021)

\begin{abstract}
Composite Higgs models must exhibit very different dynamics from quantum chromodynamics (QCD) regardless whether they describe the Higgs boson as a dilatonlike state or a pseudo-Nambu-Goldstone boson. Large separation of scales and large anomalous dimensions are frequently desired by phenomenological models. Mass-split systems are well-suited for composite Higgs models because they are governed by a conformal fixed point in the ultraviolet but are chirally broken in the infrared. In this work we use lattice field theory calculations with domain wall fermions to investigate a system with four light and six heavy flavors. We demonstrate how a nearby conformal fixed point affects the properties of the four light flavors that exhibit chiral symmetry breaking in the infrared. Specifically we describe hyperscaling of dimensionful physical quantities and determine the corresponding anomalous mass dimension. We obtain $y_{m}=1+\gamma^{*}=1.47(5)$ suggesting that $N_{f}=10$ lies inside the conformal window. Comparing the low energy spectrum to predictions of dilaton chiral perturbation theory, we observe excellent agreement which supports the expectation that the $4+6$ mass-split system exhibits near-conformal dynamics with a relatively light $0^{++}$isosinglet scalar.
\end{abstract}

DOI: $10.1103 /$ PhysRevD.103.014504

\section{INTRODUCTION}

Experiments have discovered a $125 \mathrm{GeV}$ Higgs boson [1-3] but so far, up to a few $\mathrm{TeV}$, no direct signs of physics

\footnotetext{
*Corresponding author. oliver.witzel@uni-siegen.de

${ }^{\dagger}$ Present address: Theoretische Physik 1, Naturwissenschaftlich-Technische Fakultät, Universität Siegen, 57068 Siegen, Germany.

Published by the American Physical Society under the terms of the Creative Commons Attribution 4.0 International license. Further distribution of this work must maintain attribution to the author(s) and the published article's title, journal citation, and DOI. Funded by SCOAP.
}

beyond the standard model (BSM) have been seen. The standard model (SM), however, is an effective theory, and new interactions are necessary to UV complete the Higgs sector, explain dark matter, and account for the matterantimatter asymmetry of the universe. For gauge theories describing the Higgs sector as a composite structure, experimental observations imply that a large separation of scales between the electroweak scale (IR) and new ultraviolet physics (UV) [4-11] is required. Theories with a large separation of scales part company from QCD, exhibiting a "walking" gauge coupling [12-14], and providing a dynamical mechanism for electroweak (EW) symmetry breaking. They can satisfy stringent constraints from EW precision measurements but avoid unnaturally large tuning of the Higgs mass. 
To explore theories with a large scale separation and infrared dynamics different from QCD, we employ the device of mass splitting [8,15-17], where the action has two fermion mass parameters: $\hat{m}_{h}$ and a smaller $\hat{m}_{\ell}$. The idea is to start with sufficiently many fermions to guarantee that at scales well above the fermion masses the theory exhibits infrared conformality. By assigning the masses $\hat{m}_{h}$ and $\hat{m}_{\ell}$ to the fermions, we create a system with $N_{h}$ heavy fermions and $N_{\ell}$ light fermions. The number of light fermions $N_{\ell}$ is chosen such that the light sector alone exhibits spontaneous chiral symmetry breaking. The resulting mass-split theory is governed by the conformal IRFP above the high scale set by $\hat{m}_{h}$. There the spectrum exhibits conformal hyperscaling, and the mass of the lightest isosinglet scalar $0^{++}$is expected to be comparable to the corresponding pseudoscalar mass [18,19].

In the infrared, the heavy fermions decouple, the gauge coupling runs to larger values, and chiral symmetry for the light flavors breaks spontaneously. The heavy-fermion mass $\hat{m}_{h}$ controls the separation of scales between the UV and IR [17]. Even though the low energy theory is chirally broken, its properties are significantly different from a QCD-like theory with $N_{\ell}$ fermions. In particular a light $0^{++}$state may enter the effective chiral Lagrangian, requiring the extension to dilaton chiral perturbation theory (dChPT) [20-25].

It is favorable to keep the total number of fermions $N_{f}=$ $N_{h}+N_{\ell}$ near the low end of the conformal window to achieve a large anomalous dimension. Specifically we study an $S U(3)$ gauge theory with four light and six heavy fermions in the fundamental representation. Although no consensus has been reached on the precise onset of the conformal window for $S U(3)$ gauge theories with fundamental fermions, there are indications that $N_{f}=10$ is infrared conformal [26-40]. By choosing a theory with four fermions in the light, chirally broken sector, our simulations can also directly be related to existing models extending the SM with a new strongly interacting sector [41-43]. In these models the Higgs boson is a pseudo-Nambu-Goldstone boson (pNGB) of the new strong sector $[41,42,44-46]$.

We explore this new, strongly coupled theory by performing large scale numerical lattice-field-theory simulations. The choice $N_{f}=10$ improves over a pilot study using four light and eight heavy flavors $[8,15,16,47-51]$ by being closer to the bottom of the conformal window. Also, we perform the numerical simulations using chiral domain-wall fermions (DWF) [52-55] which preserve the flavor structure. While numerically more costly, DWF provide a theoretically clean environment to perform investigations of strongly coupled theories near a conformal IR fixed point.

We briefly introduce the details of the numerical simulations before we demonstrate hyperscaling and determine the mass anomalous dimension. This allows us to explore implications for a possible effective description at low energies. Finally we give an outlook on our future calculations of phenomenologically important quantities. Preliminary results have been reported in [56,57].

\section{NUMERICAL SIMULATIONS}

Simulations are performed on hypercubic lattices using $(L / a)^{3} \times T / a$ volumes with $L / a=24$ or 32 and $T / a=$ 64 where $a$ indicates the lattice spacing. We simulate the $S U(3)$ gauge system with four light and six heavy flavors using the Symanzik gauge action [58,59] with 3-times stout-smeared ( $\rho=0.1)$ [60] Möbius domain wall fermions [55] $\left(b_{5}=1.5, c_{5}=0.5\right)$. DWF are simulated by adding a fifth dimension of extent $L_{s}$ which separates the physical modes of four dimensional space-time. For practical reasons $L_{s}$ needs to be finite i.e., DWF exhibit a small, residual chiral symmetry breaking, conventionally parametrized as an additive mass term $a m_{\text {res. }}$. In our simulations we choose $L_{s}=16$ and set the domain wall height $M_{5}=1$. We determine the residual chiral symmetry breaking
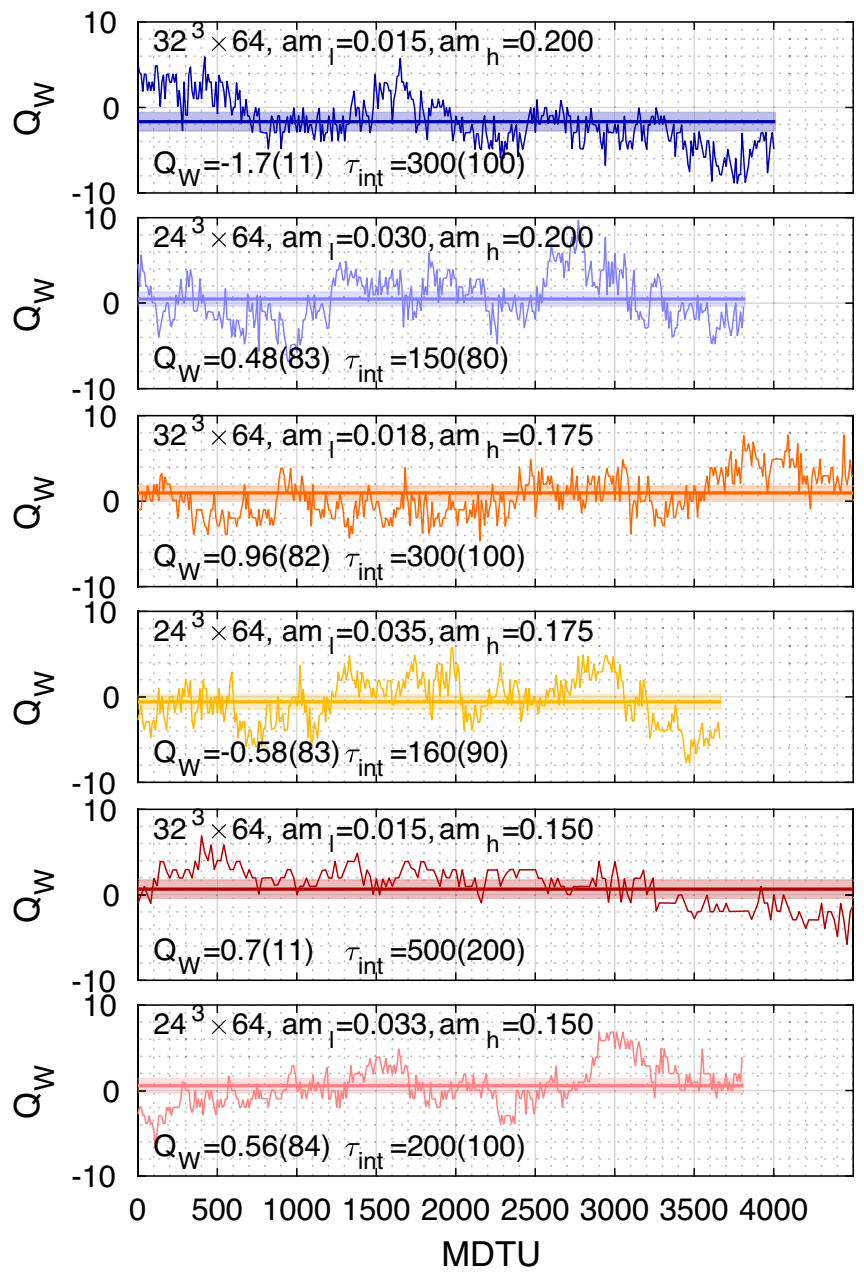

FIG. 1. Monte Carlo histories of the topological charge obtained using Wilson gradient flow at flow time $t=L^{2} / 32$ for six high statistics ensembles. The integrated autocorrelation time $\tau_{\text {int }}$ of the topological charge $Q_{W}$ is determined using the $\Gamma$-method [62] and quoted in units of MDTU. 
numerically and find small values of $O\left(10^{-3}\right)$. To correctly refer to the dimensionless lattice masses, we introduce the notation

$$
\hat{m}_{x} \equiv a \tilde{m}_{x}=a\left(m_{x}+m_{\text {res }}\right) \quad \text { with } \quad x=\ell, h .
$$

Based on insight from our accompanying step-scaling investigation [32,33], we set the bare gauge coupling $\beta=6 / g_{0}^{2}=4.03$, close to the IRFP of the underlying conformal theory with ten degenerate flavors. The hybrid Monte Carlo (HMC) update algorithm [61] with a trajectory length of $\tau=2 \mathrm{MDTU}$ (molecular dynamics time units) is used to generate ensembles of dynamical gauge field configurations with $1-3 \mathrm{k}(0.3-0.5 \mathrm{k})$ thermalized trajectories for $a m_{\ell} \leq 0.04\left(a m_{\ell}>0.04\right)$. Using input heavy flavor mass $a m_{h}=0.200,0.175$, and 0.150, we explore the $4+6$ system choosing five or seven values for the input light flavor mass in the range $0.015 \leq a m_{\ell} \leq 0.100$. Spectrum measurements are performed every 20 (10) MDTU for $a m_{\ell}<$ $0.04\left(a m_{\ell} \geq 0.04\right)$ and we decorrelate subsequent measurements by randomly choosing source positions. Remaining autocorrelations are estimated using the $\Gamma$-method [62] and accounted for by correspondingly binning subsequent measurements in our jackknife analysis. For all ensembles we observe frequent changes of the topological charge measured every $10 \mathrm{MDTU}$ (20 MDTU for $m_{\ell} / m_{h}=0.015 / 0.150$ ) which typically is a quantity exhibiting the longest autocorrelation times in the system. Examples of the Monte Carlo histories for six high statistics ensembles are shown in Fig. 1.

\section{HYPERSCALING}

To understand the properties of mass-split systems, we refer to Wilsonian renormalization group (RG). In the UV both mass parameters are much lighter than the cutoff $\Lambda_{\text {cut }}=1 / a: \hat{m}_{l} \ll 1, \hat{m}_{h} \ll 1$. As the energy scale $\mu$ is lowered from the cutoff, the RG flowed lattice action moves in the infinite parameter action space as dictated by the fixed point structure of the $N_{f}$ flavor conformal theory. The masses are increasing according to their scaling dimension $y_{m}, \hat{m}_{\ell, h} \rightarrow \hat{m}_{\ell, h}(a \mu)^{-y_{m}}$, but we assume that they are still small so the system remains close to the conformal critical surface. All other couplings are irrelevant and approach the IRFP as the energy scale is lowered.

If the gauge couplings reach the vicinity of the IRFP, only the two masses change under RG flow. We can use standard hyperscaling arguments [63-65] to show that any physical quantity $a M_{H}$ of mass dimension one follows, at leading order, the scaling form [16]

$$
a M_{H}=\hat{m}_{h}^{1 / y_{m}} \Phi_{H}\left(\hat{m}_{\ell} / \hat{m}_{h}\right),
$$

where $y_{m}=1+\gamma_{m}^{\star}$ is the universal scaling dimension of the mass at the IRFP and $\Phi_{H}$ some function of $\hat{m}_{\ell} / \hat{m}_{h}$. $\Phi_{H}$ depends on the observable $H$ and could be qualitatively

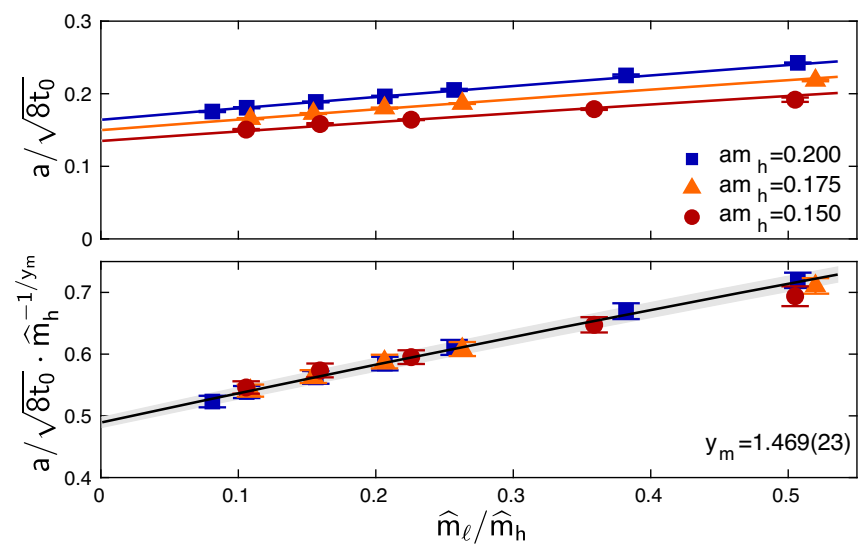

FIG. 2. The inverse Wilson flow scale $a / \sqrt{8 t_{0}}$ and interpolating fit according to Eq. (2) as function of $\hat{m}_{\ell} / \hat{m}_{h}$. The bottom panel shows "curve collapse" for $\Phi_{\sqrt{8 t_{0}}}\left(\hat{m}_{\ell} / \hat{m}_{h}\right)=a / \sqrt{8 t_{0}} \cdot \hat{m}_{h}^{-1 / y_{m}}$.

different for different $H .{ }^{1}$ The scaling relation Eq. (2) is valid as long as the gauge couplings remain at the IRFP and lattice masses are small, i.e., even in the $\hat{m}_{\ell}=0$ chiral limit. As a consequence, ratios of masses

$$
\frac{M_{H 1}}{M_{H 2}}=\frac{\Phi_{H 1}\left(\hat{m}_{\ell} / \hat{m}_{h}\right)}{\Phi_{H 2}\left(\hat{m}_{\ell} / \hat{m}_{h}\right)}
$$

depend only on $\hat{m}_{\ell} / \hat{m}_{h}$. The heavy flavors decouple when $\hat{m}_{h}(a \mu)^{-y_{m}} \approx 1$. At that point the light flavors condense and spontaneously break chiral symmetry. This allows us to define the hadronic or chiral symmetry breaking scale

$$
\Lambda_{H}=\hat{m}_{h}^{1 / y_{m}} a^{-1} .
$$

As the energy scale $\mu$ is lowered below $\Lambda_{H}$, the gauge coupling starts running again. However, properties of the IRFP are already encoded in hadronic observables. We have established hyperscaling of ratios in the $4+8$ flavor system $[8,16]$ and preliminary results for the $4+6$ system are reported in [56,57].

In Fig. 2 we illustrate hyperscaling and the determination of $y_{m}$ by considering the inverse Wilson flow scale $a / \sqrt{8 t_{0}}$ as the quantity $a M_{H}$ in Eq. (2). The dimensionful quantity $1 / \sqrt{8 t_{0}}$ is proportional to the energy scale where the renormalized running coupling in the gradient flow scheme equals a reference value $\left(g_{G F}^{2} \approx 16\right)$ [66]. The top panel shows $a / \sqrt{8 t_{0}}$ as the function of $\hat{m}_{\ell} / \hat{m}_{h}$. While the data corresponding to our three different $a m_{h}$ values are different, each set on its own follows a smooth, almost linear curve. This suggests to parametrize the unknown function $\Phi_{\sqrt{8 t_{0}}}\left(\hat{m}_{\ell} / \hat{m}_{h}\right)$ using a low-order polynomial and perform

\footnotetext{
${ }^{1}$ Equivalent to Eq. (2) is the hyperscaling relation, $a M_{H}=\hat{m}_{l}^{1 / y_{m}} \Phi_{H}\left(\hat{m}_{\ell} / \hat{m}_{h}\right)$, given in Ref. [16]. Depending on the observable and scaling test, one or the other form might be preferable.
} 


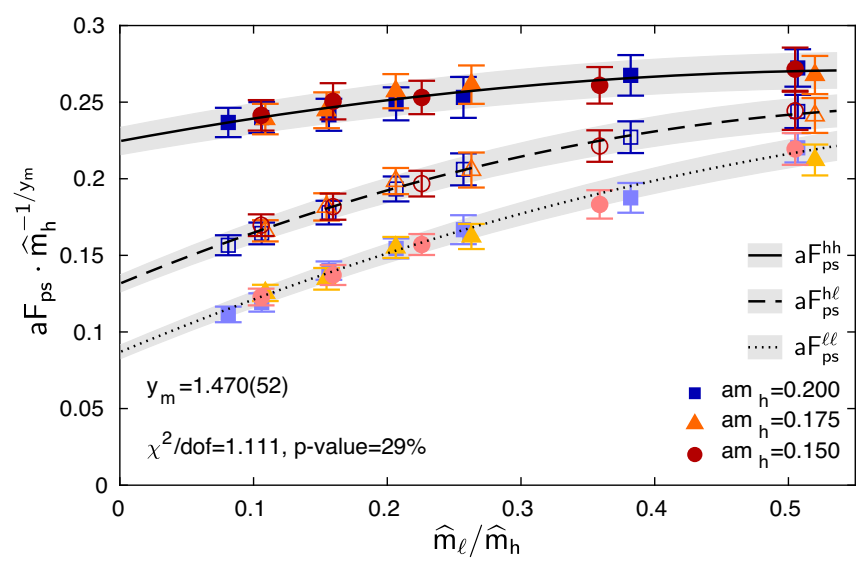

FIG. 3. Hyperscaling of the light-light (shaded symbols), heavy-light (open symbols), and heavy-heavy (filled symbols) pseudoscalar decay constant as the function of $\hat{m}_{\ell} / \hat{m}_{h}$. A combined fit based on Eq. (2) determines $y_{m}$.

a combined fit to all 17 data points in Fig. 2 using the Ansatz given in Eq. (2). A fit with a quadratic polynomial describes our data well. Small deviations of very precise $a / \sqrt{8 t_{0}}$ values lead to $\chi^{2} /$ d.o.f. $\approx 3$ and $y_{m}=1.469(23)$ with likely underestimated statistical uncertainties.

The bottom panel of Fig. 2 shows the data points for $a / \sqrt{8 t_{0}} \cdot \hat{m}_{h}^{-1 / y_{m}}$ and the quadratic fit function $\Phi_{\sqrt{8 t_{0}}}\left(\hat{m}_{\ell} / \hat{m}_{h}\right)$, exhibiting the expected "curve collapse." We find similar curve collapse for other observables and show in Fig. 3 the result for a combined, correlated fit to the light-light $(\ell \ell)$, heavy-light $(h \ell)$, and heavy-heavy $(h h)$ pseudoscalar decay constant $a F_{p s}$. Since the determination of $a F_{p s}$ is equally precise for $\ell \ell, h \ell$, or $h h$ states, this fit provides a representative determination of $y_{m}$ with a good $p$-value. Subsequently we use

$$
y_{m}=1+\gamma_{m}^{\star}=1.470(52),
$$

as our reference value and note it is consistent within uncertainties to determinations from other observables like vector or pseudoscalar masses. Further $y_{m}$ is in agreement to an independent determination based on gradient flow [67] and comparable to predictions from analytical calculations $[38,68,69]$. The predicted $\gamma_{m}^{\star}$ is substantially below 1 , the value expected for a system close to the sill of the conformal window $[12,70]$. Since dChPT analysis of the $N_{f}=8$ data [71,72] predicts $\gamma_{m}^{*}$ near 1 [20-25], this indicates the sill of the conformal window lies between $N_{f}=8$ and 10 , whereas the 12 flavor system $\left(\gamma_{m}^{\star} \approx 0.24\right.$ $[38,68,69,73-78])$ is even deeper in the conformal regime.

The scaling of $a / \sqrt{8 t_{0}}$ is particularly interesting because it shows that the lattice spacing in the $\hat{m}_{\ell}=0$ chiral limit has a simple dependence on the heavy flavor mass

$$
a=\left.\left(\hat{m}_{h}\right)^{1 / y_{m}} \cdot \Phi_{\sqrt{8 t_{0}}}(0) \cdot \sqrt{8 t_{0}}\right|_{m_{\ell}=0},
$$

where $\Phi_{\sqrt{8 t_{0}}}(0)$ is a finite number, $\approx 0.48$, in the $4+6$ system. This confirms the expectation that the continuum $a=0$ limit is approached as $\hat{m}_{h}$ decreases. Combined with Eq. (4) it predicts the hadronic scale

$$
\Lambda_{H}^{-1}=\left.\Phi \sqrt{8 t_{0}}(0) \cdot \sqrt{8 t_{0}}\right|_{m_{\ell}=0} .
$$

\section{LOW ENERGY EFFECTIVE DESCRIPTION}

In the low energy infrared limit our system exhibits spontaneous chiral symmetry breaking. It should be described by a chiral effective Lagrangian which smoothly connects to the hyperscaling relation Eq. (2), valid at the hadronic scale $\mu=\Lambda_{H}$. In order to combine datasets with different $\hat{m}_{h}$, we express the lattice scale $a$ in terms of the hadronic scale $\Lambda_{H}$

$$
M_{H} / \Lambda_{H}=\left(a M_{H}\right) \cdot \hat{m}_{h}^{-1 / y_{m}}=\Phi_{H}\left(\hat{m}_{\ell} / \hat{m}_{h}\right) .
$$

Below the hadronic scale $\Lambda_{H}$, the $4+6$ system reduces to a chirally broken $N_{f}=4$ system. The low energy effective theory (EFT) expresses the dependence of physical quantities on the running fermion mass $m_{f}$ of the light flavors. At the hadronic energy scale the light flavor mass in lattice units is $\hat{m}_{\ell}\left(a \Lambda_{H}\right)^{-y_{m}}$, predicting

$$
m_{f} \propto \hat{m}_{\ell}\left(a \Lambda_{H}\right)^{-y_{m}} \cdot \Lambda_{H}=\left(\hat{m}_{\ell} / \hat{m}_{h}\right) \cdot \Lambda_{H} .
$$

The continuum limit is taken by tuning $\hat{m}_{h} \rightarrow 0$ while keeping $\hat{m}_{\ell} / \hat{m}_{h}$ fixed.

For $\hat{m}_{\ell} / \hat{m}_{h} \lesssim 1$, we expect the $0^{++}$ground state to be dominated by the light fermions. It is confined at scales of order $\Lambda_{H}$ as are the other states, but its mass could well be small, comparable to the $\ell \ell$ pseudoscalar mass. An EFT describing the small mass regime then needs to incorporate the light scalar state together with the pseudoscalars. In the $m_{f}=0$ limit, only the pseudoscalar states are massless. The $0^{++}$decouples at very low energies and $N_{f}=4 \mathrm{ChPT}$ should describe the data.

The dChPT Lagrangian incorporates the effect of a light dilaton state [20-25]. While derived for a chirally broken system with degenerate fermions just below the conformal window, we explore its application to our near-conformal mass-split system.

$\mathrm{dChPT}$ predicts the scaling relation

$$
d_{0} \cdot F_{p s}^{2-y_{m}}=M_{p s}^{2} / m_{f},
$$

which is a general result first discussed in Refs. [20,22] and independent of the specific form of the dilaton effective potential. The quantity $d_{0}$ is a combination of low energy constants. Using Eq. (8) we express this relation in terms of lattice quantities of the light sector (dropping the superscripts $\ell \ell$ ) 

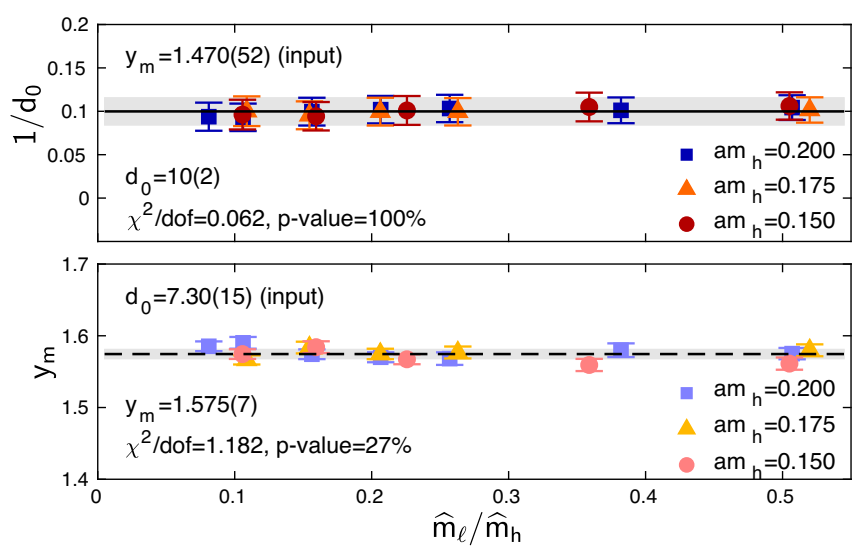

FIG. 4. Scaling test of dChPT exploiting Eq. (11). The top panel uses $y_{m}$ [Eq. (5)] as input to fit $1 / d_{0}$, the bottom panel shows a fit for $y_{m}$ from a scan over $d_{0}$ values used as input.

$$
d_{0} \cdot\left(a F_{p s}\right)^{2-y_{m}}=\left(a M_{p s}\right)^{2} / \hat{m}_{\ell} .
$$

From Eq. (2) we can deduce that $d_{0}=\left(a M_{p s}\right)^{2}$. $\left(a F_{p s}\right)^{-2+y_{m}} / \hat{m}_{\ell}$ may only depend on $\hat{m}_{\ell} / \hat{m}_{h}$, whereas Eq. (10) states $d_{0}$ is a constant.

Since our main goal is to study Eq. (10), we simply fix $y_{m}$ from Eq. (5) and determine $d_{0}$ using Eq. (11). As shown in the top panel of Fig. 4, our data form a flat line without dependence on $\hat{m}_{\ell} / \hat{m}_{h}$. A direct fit of our data to Eq. (11) to determine $y_{m}$ and $d_{0}$ simultaneously is troublesome because $a F_{p s}$ and $a M_{p s}$ have similar size uncertainties, are highly correlated, and the relation is nonlinear. Instead we perform a second test scanning a range of input values for $d_{0}$ and fit for $y_{m}$. At a minimum $\chi^{2} /$ d.o.f. we obtain a $y_{m}=1.575(7)$ within $2 \sigma$ of our reference value and shown in the lower panel of Fig. 4. In summary, our data are consistent with Eq. (11) and we obtain a rough estimate of $y_{m}$ and $d_{0}$.

Assuming a specific form of the dilaton potential leads to another dChPT relation [25]

$$
\frac{M_{p s}^{2}}{F_{p s}^{2}}=\frac{1}{y_{m} d_{1}} W_{0}\left(\frac{y_{m} d_{1}}{d_{2}} m_{f}\right)
$$

where $W_{0}$ is the Lambert W-function and $d_{1}, d_{2}$ are mass independent constants. Figure 5 shows a fit of our data to Eq. (12). The fit has an excellent $p$-value and allows us to determine the constants $d_{1}$ and $d_{2}$. Relations of $N_{f}=4$ $\mathrm{ChPT}$ at leading and next-to-leading order exhibit a mass dependence different from Eqs. (10) and (12) and do not describe our data.

Finally we comment on the mass dependence of $\sqrt{8 t_{0}} / a$. In ChPT this quantity has a linear mass dependence and corrections enter only at NNLO [79]. So far dChPT does not provide a useful description for $\sqrt{8 t_{0}} / a$ [25]. Our results in Fig. 2 show however that $a / \sqrt{8 t_{0}}$ obeys the usual

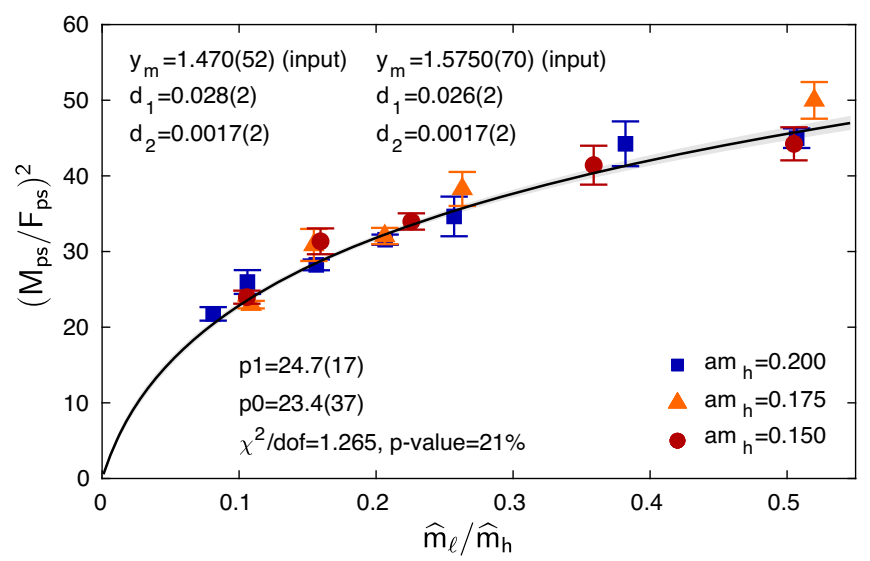

FIG. 5. Test of dChPT using Eq. (12). The black line with gray band is fitting our ratios $\left(M_{p s} / F_{p s}\right)^{2}$ to the function $y=p_{0} W_{0}\left(p_{1} \cdot \hat{m}_{\ell} / \hat{m}_{h}\right)$.

hyperscaling relation in mass-split systems and $a \sqrt{8 t_{0}}$. $\hat{m}_{h}{ }^{-1 / y_{m}}$ is well described by a linear mass dependence.

\section{CONCLUSION}

In this work we highlight the unique features of the $4+6$ mass-split system built on a conformal IRFP. We show that physical masses exhibit hyperscaling and determine the universal mass scaling dimension of the corresponding $N_{f}=10$ system $y_{m}=1+\gamma_{m}^{\star}=1.47(5)$. This value is smaller than expected for a theory near the edge of the conformal window suggesting that $N_{f}=9$ or 8 flavor models could be closer to the sill of the conformal window.

We compare our numerical results to predictions based on $\mathrm{dChPT}$ relations and find good agreement. Leading and next-to-leading order standard $N_{f}=4 \mathrm{ChPT}$ is, however, not consistent with our data. This strongly suggests that the $\mathrm{O}^{++}$isosinglet scalar of the $4+6$ mass-split system is a light state for the investigated parameter range.

There are many important questions to be studied in the future. Numerically determining the $0^{++}$scalar mass has the highest priority. Investigation of the baryonic anomalous dimension, relevant for partial compositeness, is already in progress [67]. Calculations of the $S$ parameter and the Higgs potential are planned as well. Finite temperature studies could identify phase transitions with potentially significant implications for the early universe.

\section{ACKNOWLEDGMENTS}

We are very grateful to Peter Boyle, Guido Cossu, Antonin Portelli, and Azusa Yamaguchi who develop the GRID software library $[80,81]$ providing the basis of this work and who assisted us in installing and running GRID on different architectures and computing centers. We thank Andrew Pochinsky and Sergey Syritsyn for developing QLUA [82,83] used for our measurements. The authors thank Maarten Golterman and Yigal Shamir for a critical 
reading and constructive comments on an early draft of this manuscript. R. C. B. and C. R. acknowledge United States Department of Energy (DOE) Award No. DE-SC0015845. K. C. acknowledges support from the DOE through the Computational Sciences Graduate Fellowship (DOE CSGF) through Grant No. DE-SC0019323. G. T. F. acknowledges support from DOE Award No. DESC0019061. A. D. G. is supported by SNSF Grant No. 200021_17576. A. H., E. T. N., and O. W. acknowledge support by DOE Award No. DE-SC0010005. D. S. was supported by UK Research and Innovation Future Leader Fellowship No. MR/S015418/1. P. V. acknowledges the support of the DOE under contract No. DE-AC5207NA27344 (LLNL). We thank the Lawrence Livermore National Laboratory (LLNL) Multiprogrammatic and Institutional Computing program for Grand Challenge supercomputing allocations. We also thank Argonne
Leadership Computing Facility for allocations through the INCITE program. A. L.C.F. is supported by DOE Contract No. DE-AC02-06CH11357. Computations for this work were carried out in part on facilities of the USQCD Collaboration, which are funded by the Office of Science of the U.S. Department of Energy, the RMACC Summit supercomputer [84], which is supported by the National Science Foundation (Awards No. ACI-1532235 and No. ACI-1532236), the University of Colorado Boulder, and Colorado State University and on Boston University computers at the MGHPCC, in part funded by the National Science Foundation (Award No. OCI1229059). We thank ANL, BNL, Fermilab, Jefferson Lab, MGHPCC, LLNL, the NSF, the University of Colorado Boulder, and the U.S. DOE for providing the facilities essential for the completion of this work.
[1] G. Aad et al. (ATLAS Collaboration), Phys. Lett. B 716, 1 (2012).

[2] S. Chatrchyan et al. (CMS Collaboration), Phys. Lett. B 716, 30 (2012).

[3] G. Aad et al. (ATLAS and CMS Collaborations), Phys. Rev. Lett. 114, 191803 (2015).

[4] R. Contino, Proc. TASI 09, 235 (2011).

[5] M. A. Luty and T. Okui, J. High Energy Phys. 09 (2006) 070 .

[6] D. D. Dietrich and F. Sannino, Phys. Rev. D 75, 085018 (2007).

[7] M. A. Luty, J. High Energy Phys. 04 (2009) 050.

[8] R. C. Brower, A. Hasenfratz, C. Rebbi, E. Weinberg, and O. Witzel, Phys. Rev. D 93, 075028 (2016).

[9] C. Csaki, C. Grojean, and J. Terning, Rev. Mod. Phys. 88, 045001 (2016).

[10] N. Arkani-Hamed, R. T. D’Agnolo, M. Low, and D. Pinner, J. High Energy Phys. 11 (2016) 082.

[11] O. Witzel, Proc. Sci., LATTICE2018 (2019) 006.

[12] K. Yamawaki, M. Bando, and K.-i. Matumoto, Phys. Rev. Lett. 56, 1335 (1986).

[13] T. W. Appelquist, D. Karabali, and L. Wijewardhana, Phys. Rev. Lett. 57, 957 (1986).

[14] M. Bando, T. Kugo, and K. Yamawaki, Phys. Rep. 164, 217 (1988).

[15] R. C. Brower, A. Hasenfratz, C. Rebbi, E. Weinberg, and O. Witzel, J. Exp. Theor. Phys. 120, 423 (2015).

[16] A. Hasenfratz, C. Rebbi, and O. Witzel, Phys. Lett. B 773, 86 (2017).

[17] A. Hasenfratz, C. Rebbi, and O. Witzel, EPJ Web Conf. 175, 08007 (2018).

[18] V. A. Miransky, Phys. Rev. D 59, 105003 (1999).

[19] Y. Aoki, T. Aoyama, M. Kurachi, T. Maskawa, K.-i. Nagai, H. Ohki, E. Rinaldi, A. Shibata, K. Yamawaki, and
T. Yamazaki (LatKMI Collaboration), Phys. Rev. Lett. 111, 162001 (2013).

[20] M. Golterman and Y. Shamir, Phys. Rev. D 94, 054502 (2016).

[21] T. Appelquist, J. Ingoldby, and M. Piai, J. High Energy Phys. 03 (2018) 039.

[22] T. Appelquist, J. Ingoldby, and M. Piai, J. High Energy Phys. 07 (2017) 035.

[23] M. Golterman and Y. Shamir, Phys. Rev. D 98, 056025 (2018).

[24] T. Appelquist, J. Ingoldby, and M. Piai, Phys. Rev. D 101, 075025 (2020).

[25] M. Golterman, E. T. Neil, and Y. Shamir, Phys. Rev. D 102, 034515 (2020).

[26] M. Hayakawa, K.-I. Ishikawa, Y. Osaki, S. Takeda, S. Uno, and N. Yamada, Phys. Rev. D 83, 074509 (2011).

[27] T. Appelquist et al., arXiv:1204.6000.

[28] T.-W. Chiu, arXiv:1603.08854.

[29] T.-W. Chiu, Proc. Sci., LATTICE2016 (2017) 228.

[30] T.-W. Chiu, Phys. Rev. D 99, 014507 (2019).

[31] Z. Fodor, K. Holland, J. Kuti, D. Nogradi, and C. H. Wong, Phys. Lett. B 779, 230 (2018).

[32] A. Hasenfratz, C. Rebbi, and O. Witzel, Phys. Lett. B 798, 134937 (2019).

[33] A. Hasenfratz, C. Rebbi, and O. Witzel, Phys. Rev. D 101, 114508 (2020).

[34] P. A. Baikov, K. G. Chetyrkin, and J. H. Kühn, Phys. Rev. Lett. 118, 082002 (2017).

[35] T. A. Ryttov and R. Shrock, Phys. Rev. D 83, 056011 (2011).

[36] T. A. Ryttov and R. Shrock, Phys. Rev. D 94, 105015 (2016).

[37] T. A. Ryttov and R. Shrock, Phys. Rev. D 94, 125005 (2016). 
[38] T. A. Ryttov and R. Shrock, Phys. Rev. D 95, 105004 (2017).

[39] O. Antipin, A. Maiezza, and J. C. Vasquez, Nucl. Phys. B941, 72 (2019).

[40] L. Di Pietro and M. Serone, J. High Energy Phys. 07 (2020) 049.

[41] T. Ma and G. Cacciapaglia, J. High Energy Phys. 03 (2016) 211.

[42] D. Buarque Franzosi, G. Cacciapaglia, and A. Deandrea, Eur. Phys. J. C 80, 28 (2020).

[43] D. Marzocca, J. High Energy Phys. 07 (2018) 121.

[44] L. Vecchi, J. High Energy Phys. 02 (2017) 094.

[45] G. Ferretti and D. Karateev, J. High Energy Phys. 03 (2014) 077.

[46] G. Ferretti, J. High Energy Phys. 06 (2016) 107.

[47] R. C. Brower, A. Hasenfratz, C. Rebbi, E. Weinberg, and O. Witzel, Proc. Sci., LATTICE2014 (2014) 254 [arXiv: 1411.3243].

[48] E. Weinberg, R. C. Brower, A. Hasenfratz, C. Rebbi, and O. Witzel, J. Phys. Conf. Ser. 640, 012055 (2015).

[49] A. Hasenfratz, R. C. Brower, C. Rebbi, E. Weinberg, and O. Witzel, Int. J. Mod. Phys. A 32, 1747003 (2017).

[50] A. Hasenfratz, C. Rebbi, and O. Witzel, Proc. Sci., LATTICE2016 (2016) 226 [arXiv:1611.07427].

[51] A. Hasenfratz, C. Rebbi, and O. Witzel, Proc. Sci., EPSHEP2017 (2017) 356.

[52] D. B. Kaplan, Phys. Lett. B 288, 342 (1992).

[53] Y. Shamir, Nucl. Phys. B406, 90 (1993).

[54] V. Furman and Y. Shamir, Nucl. Phys. B439, 54 (1995).

[55] R. C. Brower, H. Neff, and K. Orginos, Comput. Phys. Commun. 220, 1 (2017).

[56] O. Witzel, A. Hasenfratz, and C. Rebbi, arXiv:1810.01850.

[57] O. Witzel and A. Hasenfratz, Proc. Sci., LATTICE2019 (2019) 115 [arXiv:1912.12255].

[58] M. Lüscher and P. Weisz, Commun. Math. Phys. 97, 59 (1985); 98, 433(E) (1985).

[59] M. Lüscher and P. Weisz, Phys. Lett. 158B, 250 (1985).

[60] C. Morningstar and M. J. Peardon, Phys. Rev. D 69, 054501 (2004).

[61] S. Duane, A. Kennedy, B. Pendleton, and D. Roweth, Phys. Lett. B 195, 216 (1987).

[62] U. Wolff (ALPHA Collaboration), Comput. Phys. Commun. 156, 143 (2004).
[63] T. DeGrand and A. Hasenfratz, Phys. Rev. D 80, 034506 (2009).

[64] L. Del Debbio and R. Zwicky, Phys. Rev. D 82, 014502 (2010).

[65] L. Del Debbio and R. Zwicky, Phys. Lett. B 700, 217 (2011).

[66] M. Lüscher, J. High Energy Phys. 08 (2010) 071.

[67] A. Hasenfratz and O. Witzel (to be published).

[68] T. A. Ryttov and R. Shrock, Phys. Rev. D 94, 105014 (2016).

[69] T. A. Ryttov and R. Shrock, Phys. Rev. D 97, 025004 (2018).

[70] S. Matsuzaki and K. Yamawaki, Phys. Rev. Lett. 113, 082002 (2014).

[71] T. Appelquist, R. Brower, G. Fleming, A. Hasenfratz, X.-Y. Jin, J. Kiskis, E. Neil, J. Osborn, C. Rebbi, E. Rinaldi, D. Schaich, P. Vranas, E. Weinberg, and O. Witzel (Lattice Strong Dynamics), Phys. Rev. D 93, 114514 (2016).

[72] T. Appelquist, R. Brower, G. Fleming, A. Gasbarro, A. Hasenfratz, X.-Y. Jin, E. Neil, J. Osborn, C. Rebbi, E. Rinaldi, D. Schaich, P. Vranas, E. Weinberg, and O. Witzel (Lattice Strong Dynamics), Phys. Rev. D 99, 014509 (2019).

[73] T. Appelquist, G. Fleming, M. Lin, E. Neil, and D. Schaich, Phys. Rev. D 84, 054501 (2011).

[74] T. DeGrand, Phys. Rev. D 84, 116901 (2011).

[75] A. Cheng, A. Hasenfratz, G. Petropoulos, and D. Schaich, J. High Energy Phys. 07 (2013) 061.

[76] A. Cheng, A. Hasenfratz, Y. Liu, G. Petropoulos, and D. Schaich, Phys. Rev. D 90, 014509 (2014).

[77] M. P. Lombardo, K. Miura, T. J. N. da Silva, and E. Pallante, J. High Energy Phys. 12 (2014) 183.

[78] Z. Li and D. Poland, arXiv:2005.01721.

[79] O. Bär and M. Golterman, Phys. Rev. D 89, 034505 (2014); 89, 099905(E) (2014).

[80] https://github.com/paboyle/Grid.

[81] P. Boyle, A. Yamaguchi, G. Cossu, and A. Portelli, Proc. Sci., LATTICE2015 (2015) 023.

[82] https://usqcd.Ins.mit.edu/w/index.php/QLUA.

[83] A. Pochinsky, Proc. Sci., LATTICE2008 (2008) 040.

[84] J. Anderson, P. J. Burns, D. Milroy, P. Ruprecht, T. Hauser, and H. J. Siegel, Deploying RMACC Summit: An HPC Resource for the Rocky Mountain Region, PEARC17 (Association for Computing Machinery, New York, 2017), pp. 1-7, https://doi.org/10.1145/3093338.3093379. 